\title{
Pathomorphological characteristics of Dupuytren's contracture in a patient with liver disease (case report)
}

\author{
N.A. Shchudlo, M.M. Shchudlo, T.A. Stupina, T.N. Varsegova, N.S. Migalkin, N.G. Shikhaleva, \\ V.V. Kostin
}

Russian Ilizarov Scientific Center for Restorative Traumatology and Orthopaedics, Kurgan, Russian Federation

\begin{abstract}
Objective To detect pathomorphological characteristics of the palmar aponeurosis in Dupuytren's contracture in patients with liver disease. Material and methods The review included a medical record and histological findings of surgical specimen of a 60-year-old patient who underwent surgeries for Dupuytren's contracture in 2016 and 2019 first on the right and then on the left side. Micropreparations of palmar aponeurosis stained with hematoxylin and eosin, Masson's trichrome, using van Gieson and Hart methods were examined with light microscopy. Results Typical lamellar pattern in thickened pretendinous cord of the palmar aponeurosis was observed in surgical specimen of 2016 with flexion contracture at the metacarpophalangeal joints in IV-V digits of the right hand that developed 4 years after the first clinical signs of the palmar fascial fibromatosis. In 2019 the patient developed grade III-IV Dupuytren's contracture at the metacarpophalangeal joints and the proximal interphalangeal joints in IV-V digits of the left hand. Active proliferative areas were observed in central parts of tendon resembling bands of digital fasciae with signs of fibrocartilaginous metaplasia and lipomatosis seen in the surgical specimen. Collagen fiber bundles were shown to intensively develop de novo at the periphery of the cords. Discussion Fibrocartilaginous metaplasia of dense Dupuytren's connective tissue was described in single publications, and this is the first report on lipomatosis and collagen fiber bundles developed de novo. Conclusion Specific features of pathomorphology of fascial fibromatosis were likely to be associated with chronic hepatitis of unknown etiology.
\end{abstract}

Keywords: Dupuytren's contracture, histological examination, fibrocartilaginous metaplasia, lipomatosis, chronic hepatitis

Dupuytren's disease is an irreversible progressive flexion deformity of the fingers due to fibromatosis of the palmar and digital fasciae. Although the condition is common among patients of hand surgery clinics the aetiopathogenesis has not been established, and the condition is believed to be hereditary and have a positive correlation with microangiopathy, changes in production of growth factors and cytokines, with hypertension being the most common comorbid disease [1]. Dupuytren's disease has many recognized associations. Diabetes mellitus, smoking, excessive alcohol intake and palm irritation are independent risk factors for disease development [2]. Meta-analysis of 33 studies showed a strong association between Dupuytren's contracture and liver disease [3]. Dupuytren's disease was found to be equally common in patients with alcoholic and non-alcoholic biopsy-proven liver disease (25\% versus $28 \%$ ) [4]. Reasons of the correlations are unknown. There are no data on specific pathomorphological characteristics of the palmar fascial fibromatosis in patients with Dupuytren's contracture and liver disease.

Objective The purpose of the study was to analyze a correlation between bilateral Dupuytren's disease and chronic hepatitis of the unknown origin.

\section{MATERIAL AND METHODS}

Between January 2014 and March 2019261 patients with Dupuytren's disease underwent surgical treatment at the Russian Ilizarov Scientific Center for Restorative Traumatology and Orthopaedics. From them, 14 patients (5.36 \%) had liver disease: two were diagnosed with chronic hepatitis of unknown etiology and 12 with serologically proven chronic hepatitis A, B or C viruses. Patients with comorbidities, familial Dupuytren's history, heavy smoking habits and alcohol users were excluded from the study $(\mathrm{n}=13)$. One patient who had none of the risk factors but liver disease was included in the study. The study was performed according to ethical principles of the Declaration of Helsinki of 1975 as revised in 2008 and approved by Institutional Review Board (Protocol № 2 (57) dtd 19.03.2018).

A fragment of palmar aponeurosis excised intraoperatively during selective fasciectomy was fixed in formalin for 3-5 days and embedded in paraffin blocks using routine technique. Longitudinal and transverse sections of 5-7 mcm thick were cut with Reichert microtome (Austria). Sections were

@ Shchudlo N.A., Shchudlo M.M., Stupina T.A., Varsegova T.N., Migalkin N.S., Shikhaleva N.G., Kostin V.V. Pathomorphological characteristics of Dupuytren's contracture in a patient with liver disease (case report). Genij Ortopedii, 2019, vol. 25, no 4, pp. 576579. DOI 10.18019/1028-4427-2019-25-4-576-579. (In Russian) 
stained with hematoxylin and eosin, Masson's trichrome, van Gieson's picrofuchsin and Hart's orcein. Images of micropreparations were acquired with AxioCam digital camera and AxioScope. A1 microscope (Carl Zeiss MicroImaging GmbH, Germany).

\section{RESULTS}

Seven years ago a 60-year-old patient noted hard nodules under the skin of both palms at rays IV-V that developed into bands 5 years ago. Limited extension of metacarpophalangeal joints of IV-V fingers on the right side followed by flexion contracture grade II occurred 4 years after the onset of the disease. Preoperative laboratory examination showed elevated liver enzymes: AST, 59; ALT, $60 \mathrm{IU} / \mathrm{mL}$ and bilirubin, $25.4 \mathrm{mkM} / \mathrm{L}$, and negative serological tests. He was seen by gastroenterologist who diagnosed the patient with NOS chronic hepatitis and administered Phosphogliv 2 capsules to be taken 3 times daily for 3 months.

Surgical intervention performed on September 30, 2016 included selective fasciectomy with the excision of pathological tissue of the palmar aponeurosis. Postoperative treatments included physical therapy, dynamic hand splinting so that the deformity was corrected and function of the right hand restored. Examination of the pretendinous cord as the surgical specimen showed predominance of fibrous cellular tissue forming tendon resembling bands or some coils, and being spread between fat lobules in some areas. Fat lobules of the hypoderma contained hypertrophied adipocytes (Fig. 1a). Sweat gland and sweat ducts were also seen (Fig. 1b) in the specimen. Cells having round or oval nuclei and scanty cytoplasm, fusiform cells with hollow oval nuclei and expressed cytoplasm, tight cellular pairs or isogenic groups were observed in tendon resembling bands (Fig. 1c). Almost no elastic fibers were detected in the pretendinous cord with orcein staining (Fig. 1d) but they were seen as part of a vessel. Avascular portions of the pretendinous cord alternated with hypervascularized areas (Fig. 1e). Scanty fibroblasts and collagen deposits were visualized around the vessels of the microvasculature. Lymphocytes infiltrating the walls of minor arteries were encountered (Fig. 1f).

In 2017 (5 years from the onset of the disease) the patient developed flexion alignment of the hand. In 2019 he was seen at the RISC "RTO" again and diagnosed with grade III-IV Dupuytren's contracture of the metacarpophalangeal (MCP) and the proximal interphalangeal (PIP) joints of the IV-V digits of the left hand. Preoperative blood tests showed normal AST (34 IU/mL), slightly increased ALT (42 IU/mL) and elevated bilirubin level $(31.5 \mathrm{mkM} / \mathrm{L})$. Surgical procedure performed on February 11, 2019 included repair of the palmar and digital fascia with the excision of pathological tissues at rays IV-V, arthrolysis of the PIP joints, transosseous fixation with the Ilizarov miniapparatus in accordanceto the principles described earlier. The surgical specimen demonstrated predominant bands of tense connective tissue (Fig. 2a), areas of lipomatosis (Fig. 2b) and multiple small collagen bundles at the periphery of thick collagen clusters (Fig. 2c).
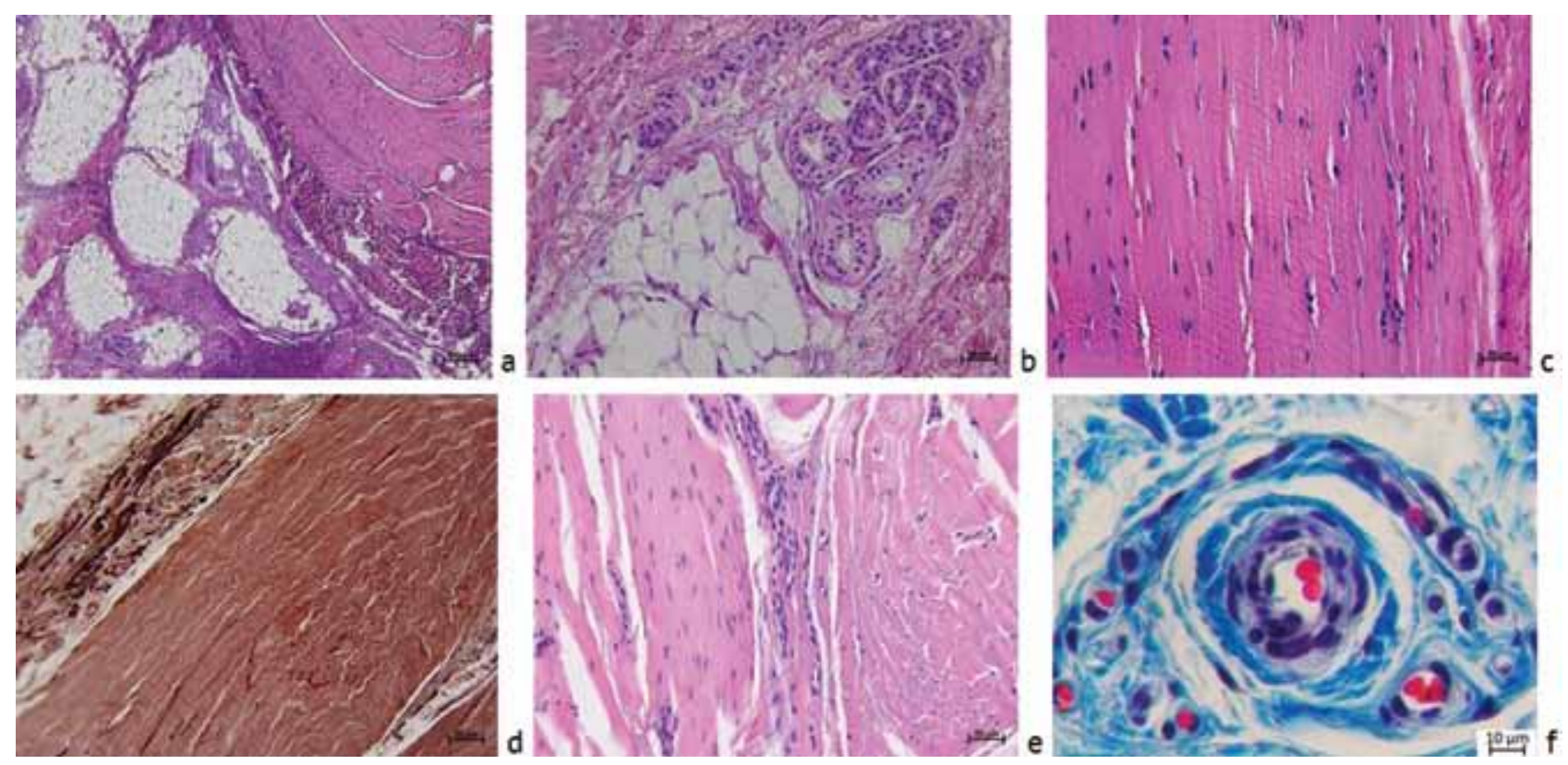

Fig. 1 Fragments of paraffin sections of the palmar aponeurosis in patient with Dupuytren's contracture and liver disease showing (a) hypertrophied adipocytes of the hypoderma; $(\boldsymbol{b})$ sweat glands; $(\boldsymbol{c}, \boldsymbol{d}, \boldsymbol{e})$ the pretendinous cord; $(\boldsymbol{f})$ minor artery. Stained with hematoxylin and eosin $(\boldsymbol{a}, \boldsymbol{b}, \boldsymbol{c}, \boldsymbol{e})$, Masson's trichrome $(\boldsymbol{f})$, orcein $(\boldsymbol{d})$. Ob. - 4, ок. - $10(\boldsymbol{a})$; оb. - 20, ок. $-10(\boldsymbol{b}, \boldsymbol{c}, \boldsymbol{d}, \boldsymbol{e})$; оb. -40 , ок. $-10(\boldsymbol{f})$ 
Fat lobules and hypertrophied adipocytes could be visualized between the bundles of collagen fibers (Fig. 2d). Masson's trichrome could reveal aberrant staining of collagen fibers (Fig. 2e,f). A greater microscope magnification showed alternating cellfree areas of dense connective tissues and cellular bands (Fig. 2g) evolved from fibroblasts/fibrocytes and myofibroblasts, as well as from grouped cartilaginous cells. Proliferative centers were encountered in cords of connective tissue in the digital fascia (Fig. $2 \mathrm{~h}$ ). The majority of nerve trunks of the palmar aponeurosis had thickened fibrous perineurium, and areas of ischemic necrosis and Wallerian degeneration were seen in endoneurium (Fig. 2i).
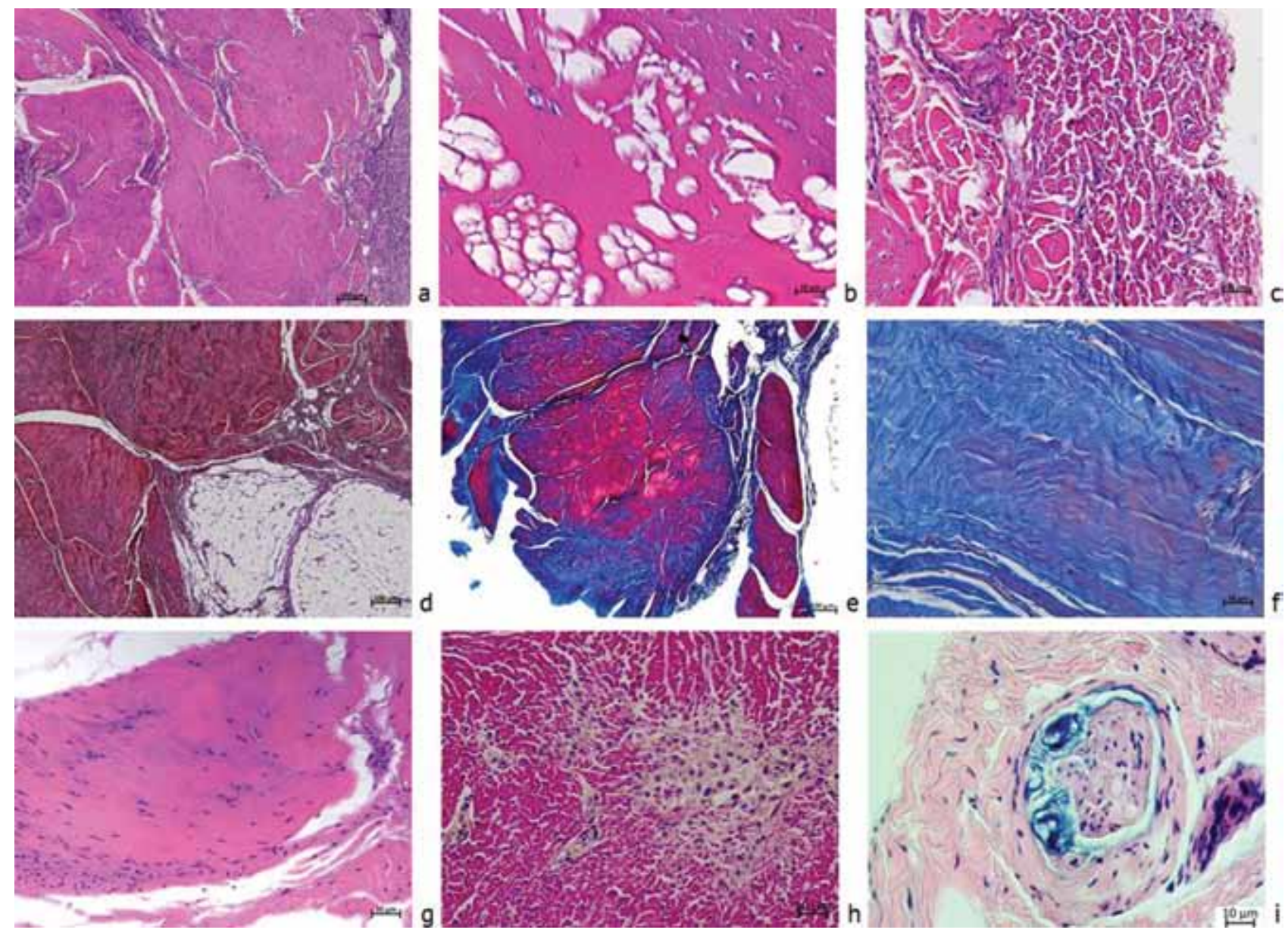

Fig. 2 Fragments of paraffin sections of the palmar aponeurosis in patient with Dupuytren's contracture and liver disease showing (a) predominant bands of the tense connective tissue; $(\boldsymbol{b})$ areas of lipomatosis; (c) small bundles of collagen fibers; $(\boldsymbol{d})$ hypertrophied adipocytes; $(\boldsymbol{e})$ aberrant staining of collagen fibers; $(\boldsymbol{f})$ cell-free areas of tense connective tissue; $(\boldsymbol{g})$ cellular bands; $(\boldsymbol{h})$ proliferative fascial center; $(\boldsymbol{i})$ destruction of the nerve trunk. Stained with hematoxylin and eosin $(\boldsymbol{a}, \boldsymbol{b}, \boldsymbol{c}, \boldsymbol{g}, \boldsymbol{i})$, Masson's trichrome $(\boldsymbol{e}, \boldsymbol{f})$, van Gieson's picrofuchsin $(\boldsymbol{d}, \boldsymbol{h})$. Ob. - 4, ок. - $10(\boldsymbol{a}, \boldsymbol{d}, \boldsymbol{e})$; оb. - 20, ок. - $10(\boldsymbol{c}, \boldsymbol{f}, \boldsymbol{g})$; оb. - 40, ок. - $10(\boldsymbol{b}, \boldsymbol{h}, \boldsymbol{i})$

\section{DISCUSSION}

Data from medical records and histological findings of the surgical specimen of the patient with bilateral Dupuytren's contracture and liver disease of unknown etiology allowed comparison of clinical and pathomorphological characteristics of the palmar fascial fibromatosis at different stages of the disease. The first surgery was produced to address grade II flexion contracture of the MCP joints of the IV-V digits of the right hand that developed 4 years after the first clinical manifestations of the palmar fascial fibromatosis. Histological assessment demonstrated typical lamellar type [6] of the thickened pretendinous band of the palmar aponeurosis and neural and vascular changes being typical for the palmar fascial fibromatosis [7].

The second surgery was performed on the left hand to address grade III-IV Dupuytren's contracture of the MCP and the PIP joints of the IV-V digits that developed 7 years after the first clinical manifestations of the fibromatosis and 2 years after the first signs of limited extension. Many authors reported less cellularity and more evident fibrosis at late stages of fibromatosis. Surgical specimen showed considerable differences in the characteristics of the palmar and digital fascia. Active proliferative centers were observed in the central portions of tendon resembling bands of the digital fascia. 
H. Millesi [8] suggests that the pathological Dupuytren's tissue arises through the transformation of existing fibers and fiber bundles and not by neoformation. The patient exhibited intense formation of collagen fibers de novo with new bundles located at the periphery of major cords. Fibrocartilaginous metaplasia in connective tissue and heterotopic ossification was reported in a case of Dupuytren's disease [9]. No reports could be found on lipomatosis that was evident in the reported case along with fibrocartilaginous metaplasia.

\section{CONCLUSION}

The specific pathomorphologic features of fascial fibromatosis were likely to be associated with comorbid condition, chronic hepatitis of unknown etiology exhibiting mild symptoms of hepatic inflammation and disturbed liver function. Further research is needed to gain a deeper insight into the diseases.
Funding This work was supported by Ministry of Health of the Russian Federation under the government research and development project to be implemented at the Russian Ilizarov Scientific Center for Restorative Traumatology and Orthopaedics in 2018-2020.

\section{REFERENCES}

1. Mansur H.G., Oliveira E.R., Gonçalves C.B. Epidemiological analysis of patients with Dupuytren's disease. Rev. Bras. Ortop., 2017, vol.53, no. 1, pp. 10-14. DOI: 10.1016/j.rboe.2017.12.003.

2. Ryhänen J., Forsman M. Dupuytren's contracture. Duodecim., 2012, vol. 128, no. 4, pp. 421-429.

3. Broekstra D.C., Groen H., Molenkamp S., Werker P.M.N., Van den Heuvel E.R. A Systematic Review and Meta-Analysis on the Strength and Consistency of the Associations between Dupuytren Disease and Diabetes Mellitus, Liver Disease, and Epilepsy. Plast. Reconstr. Surg., 2018, vol. 141, no. 3, pp. 367e-379e. DOI: 10.1097/PRS.0000000000004120.

4. Houghton S., Holdstock G., Cockerell R., Wright R. Dupuytren's contracture, chronic liver disease and IgA immune complexes. Liver, 1983, vol. 3, no. 4, pp. 220-224.

5. Dolganova T.I., Shchudlo N.A., Shabalin D.A., Kostin V.V. Otsenka gemodinamiki arterii kisti i mikrotsirkuliatsii kozhi pri kontrakture Diupiuitrena 3-4 stadii do i posle operativnogo lecheniia s primeneniem chreskostnoi fiksatsii po G.A. Ilizarovu [Assessment of hemodynamics of the hand arteries and skin microcirculation in Dupuytren's contracture stages 3 to 4 of before and after surgical treatment with the use of Ilizarov transosseous fixation]. Genij Ortopedii, 2019, vol. 25, no. 1, pp. 86-92. (in Russian)

6. Shchudlo N.A., Kostin V.V. Patogenez neiropatii pri kontrakture Diupiuitrena [Pathogenesis of neuropathy in Dupuytren's contracture]. Genij Ortopedii, 2019, vol. 25, no. 1, pp. 58-64. (in Russian)

7. Nezelof C. Histological aspects of Dupuytren's contracture. In: Hueston J.T., Tubiana R., eds. Dupuytren's Disease. London: Grune \& Stratton, 1974, pp. 25-27.

8. Millesi H. The clinical and morphological course of Dupuytren's disease. In: Hueston J.T., Tubiana R., eds. Dupuytren's Disease. London: Grune \& Stratton, 1974, pp. 49-60.

9. Sadideen H., Athanasou N., Ashmore A., McNab I. Heterotopic ossification in Dupuytren's disease: clinical and histological significance. J. Bone Joint Surg. Br., 2011, vol. 93, no. 12, pp. 1676-1678. DOI: 10.1302/0301-620X.93B12.27438.

Received: 29.05.2019

\section{Information about the authors:}

1. Natalia A. Shchudlo, M.D., Ph.D.,

Russian Ilizarov Scientific Center for Restorative Traumatology and Orthopaedics, Kurgan, Russian Federation, Email: nshchudlo@mail.ru

2.Mikhail M. Shchudlo, M.D., Ph.D.,

Russian Ilizarov Scientific Center for Restorative Traumatology and Orthopaedics, Kurgan, Russian Federation

3. Tatyana A. Stupina, Ph.D. of Biological Sciences,

Russian Ilizarov Scientific Center for Restorative Traumatology and Orthopaedics, Kurgan, Russian Federation, Email: StupinaSTA@mail.ru

4. Tatyana N. Varsegova, Ph.D. of Biological Sciences,

Russian Ilizarov Scientific Center for Restorative Traumatology and Orthopaedics, Kurgan, Russian Federation, Email: varstn@mail.ru

5. Nikolai S. Migalkin,

Russian Ilizarov Scientific Center for Restorative Traumatology and Orthopaedics, Kurgan, Russian Federation

6. Natalia G. Shikhaleva, M.D., Ph.D.,

Russian Ilizarov Scientific Center for Restorative Traumatology and Orthopaedics, Kurgan, Russian Federation

7. Vadim V. Kostin, M.D.,

Russian Ilizarov Scientific Center for Restorative Traumatology and Orthopaedics, Kurgan, Russian Federation 